\title{
耐受偏二甲肼的芦苇变异株系的篮选
}

\author{
杨树德 ${ }^{1}$ 徐文忠 ${ }^{1}$ 刘士锐 ${ }^{2}$ 张 统 ${ }^{2}$ 麻 密 ${ }^{\text {* }}$ \\ (1 中国科学院植物研究所光合作用与环境分子生理学重点实验室, 北京 100093) \\ （2 北京特种工程设计院, 北京 100028）
}

\begin{abstract}
摘 要 四氧化二氮/偏二甲肼 (Unsymmetrical dimethyl-hybrazine, 简称 UDMH) 为常用的航天器双组元液体推进剂。 偏二甲肼沸点低, 具有 “三致”毒性, 可在使用过程中释放到环境中, 对污染地的生物多样性和人类健康构成威胁, 因此迫切需要清除环境中的偏二甲肼。该文采用细胞工程的技术手段, 以芦苇幼苗的下肧轴为材料诱导愈伤组 织, 继而通过逐步提高笚选压力选育出可耐受偏二甲肼的变异细胞系, 再诱导变异细胞系分化, 为可治理含偏二甲 肼的废水的人工湿地处理系统的构建提供理想的工程植物。结果表明: 芦苇的愈伤组织在含有 $0.5 \mathrm{mg} \cdot \mathrm{L}^{-1} 2,4-\mathrm{D}$ 的 MS 培养基上生长良好; 偏二甲肼对该愈伤组织生长的半致死剂量为 $16.3 \mathrm{mmol} \cdot \mathrm{L}^{-1}$; 在分别含有 1.63 .3 .26 和 $8.15 \mathrm{mmol} \cdot \mathrm{L}^{-1}$ 偏二甲肼的篮选培养基上进行 $3 \sim 6$ 次继代培养后, 可得到较稳定的抗性细胞系, 培养 $23 \mathrm{~d}$ 后的相对 生长量分别为对照的 $90.4 \% 、 84.3 \%$ 和 $43.4 \%$, 培养 $43 \mathrm{~d}$ 后的相对生长量分别为对照的 $95.6 \% 、 91.7 \%$ 和 $46.8 \%$; 但是只有前两类抗性细胞系可在含 $0.1 \mathrm{~g} \bullet \mathrm{L}^{-1} \mathrm{KT} 、 0.01 \mathrm{~g} \bullet \mathrm{L}^{-1} \mathrm{NAA}$ 和相应浓度的偏二甲肼的 $\mathrm{MS}$ 培养基中诱导分 化; 将绿色再生苗转移到不含激素但含偏二甲肼的培养基上强化根的生长, 再经过 $35 \mathrm{~d}$ 左右的适应性驯化, $70 \%$ 以 上的再生苗可成功地转移至温室中培养, 为日后人工湿地系统的构建奠定了基础。
\end{abstract}

关键词 偏二甲肼 芦苇 体外篮选 变异细胞系 细胞工程

\section{SELECTION OF UDMH TOLERANT VARIANT LINES OF AN AQUATIC REED}

\author{
YANG Shu-De $^{1} \quad$ XU Wen-Zhong ${ }^{1} \quad$ LIU Shi-Rui ${ }^{2} \quad$ ZHANG-Tong ${ }^{2}$ and MA-Mi ${ }^{1}$ * \\ (1 Key Laboratory of Photosynthesis and Environmental Molecular Physiology, Institute of Botany, Chinese Academy of Sciences, Beijing 100093, China) \\ (2 Beijing Special Engineering Design and Research Institution, Beijing 100028, China)
}

\begin{abstract}
Unsymmetrical dimethyl-hydrazine (UDMH) is a highly volatile and reactive compound widely used as a liquid rocket propellant fuel in manned and unmanned space and missile systems. Exposure to UDMH is an important occupational health issue, which will increase in significance as space applications increase. Several methods have been tested for removing UDMH from the environment, such as catalytic oxidation, active carbon absorption and ion exchange resins. However, little attention has been given to the application of constructed wetlands to remove UDMH from groundwater even though constructed wetlands are a promising measure for wastewater treatment.

In expectation of constructing of an effective wetland by the variant reed lines to deal with UDMH polluted groundwater, attempts were made to screen for UDMH tolerant variant lines of reeds using cellular engineering technology in this study.

Seeds of the reed (Phragmites communis) collected from the river banks near by a satellite-launching basement were sterilized with $70 \%$ ethanol and $2 \%$ hypochlorous acid and then transferred to a MS medium for germination. After germination, hypocotyl seedling segments were used to induce callus on the MS medium supplemented with $1 \mathrm{mg} \bullet \mathrm{L}^{-1}$, 4-dichlorophenoxyacetic acid. The UDMH-tolerant cell lines were obtained by continuous selection on the UDMH containing medium supplemented with $0.5 \mathrm{mg} \cdot \mathrm{L}^{-1}$ 2, 4-dichlorophenoxyacetic acid. Three levels of UDMH tolerant cell lines were screened for that contained either 1.63, 3.26, or $8.15 \mathrm{mmol} \cdot \mathrm{L}^{-1} \mathrm{UDMH}$ after $3-6$ times of subculture. Relative growth, measured at the end of a 23-day incubation, was $90.4 \%, 84.3 \%$, and $43.4 \%$ of the control in the three treatments, respectively, and, at the end of a 43-day incubation, was $95.6 \%, 91.7 \%$, and $46.8 \%$ of the control, respectively. These results showed that UDMH could inhibit the growth of the callus at $8.15 \mathrm{mmol} \cdot \mathrm{L}^{-1}$. Regeneration of plantlets from the selected cell lines was conducted on UDMH containing MS medium supplemented with $0.1 \mathrm{~g} \bullet \mathrm{L}^{-1} 6$-Benzy-
\end{abstract}


laminopurine and $0.01 \mathrm{~g} \bullet \mathrm{L}^{-1}$ 1-naphthyl acetic acid. However, only the cell lines grown in the lower two treatments could be successfully differentiated, the $8.15 \mathrm{mmol} \cdot \mathrm{L}^{-1} \mathrm{UDMH}$ failed to differentiate. These results indicated that the suppressive effect of UDMH on callus differentiation was greater than on callus growth. Plantlets screened under UDMH stress were more tolerant to UDMH than the control, which were plantlets regenerated from callus without UDMH stress. Root growth of the differentiated plantlets was enhanced by transferring them to a hormone-free MS medium supplemented with UDMH for 35 days prior to successful transplantation of the plantlets into the soil. Finally, more than $70 \%$ of regenerated plantlets could be successfully transferred to the greenhouse. Further experiments estimating the growing status and remediation efficiency in UDMH polluted water of these variant seed lines were undertaken.

Key words UDMH, Reed, In vitro selection, variant lines, Cellular engineering

四氧化二氮/偏二甲肼 ( Unsymmetrical dimethylhydrazine, 简称 UDMH) 为常用的航天器双组元液体 推进剂。偏二甲肼易挥发, 有致癌、致畸、致突变的 毒性。在推进剂咜存、运输、转注、火箭发动机试车、 火箭发射、管道及设备冲洗中产生的含有偏二甲肼 的废水可对卫星发射基地的地下水源和空气造成污 染, 对生物多样性和人的中枢神经系统、消化系统、 血液循环系统有潜在威胁 (岳茂兴等, 2004; 朱红兵 等, 2003; Zelnick et al., 2003; 孟晓红等, 2000; Frazier et al., 1991; Ernst et al., 1987), 因此迫切需要对含 有偏二甲肼的污水进行治理。近年来, 人工湿地系 统广泛应用于污水处理、地表水体污染控制等领域 （Sun et al.，1999）。利用人工湿地系统处理偏二甲 肼废水污水与传统的理化方法, 如活性炭吸附法、化 学氧化法、催化氧化法等(宫宝安, 1994; 张家得, 1993; Lunn \& Sansone，1994)相比, 具有投资低、能耗 低、生态环境效益高、污染物去除率高、减轻二次污 染等优点, 在有大量荒地的卫星发射地区具有广泛 应用前景。但目前这方面的工作尚未见报道, 可耐 受或降解偏二甲肼的植物品种的选育则是决定人工 湿地系统成功构建的一个重要环节。

芦苇( Phragmites communis) 是一个世界广布种, 可以抵抗非常恶劣的环境条件 (Ye et al., 1997), 而 且芦苇对多种污染物抗性强, 并具有一定的分解净 化能力, 因而成为利用人工湿地处理污水的首选植 物之一( Sun et al., 1999; Brix, 1989)。在长期的适 应过程中, 不同生境中生长的芦苇不仅在形态、生态 特性等方面有明显差异, 而且在生理和遗传学方面 也有深刻变异(张承烈和陈国仓, 1991)。因此,在将 芦苇应用于环境治理工程中时, 必须考虑到工程当 地的环境条件, 以选择合适的生态型。在本研究的 前期调研中发现, 芦苇是卫星发射基地所处戈壁滩 的一个典型的优势种。本文以生长在卫星发射基地 附近河岸的芦苇种子为材料, 借助细胞工程技术篮
选强耐受火箭推进剂——偏二甲肼的变异体, 希望 得到的变异株系能较多地保留当地芦苇适应严酷环 境的特性, 以取得较佳的应用效果。

\section{1 材料和方法}

\section{1 材料与试剂}

芦苇种子采自生长在酒泉卫星发射基地的河岸 边。偏二甲肼由北京特种工程设计院提供。

\section{2 愈伤组织的诱导与继代培养}

选取 100 粒芦苇种子, 用 $70 \%$ 乙醇处理 $1 \mathrm{~min}$, 再用 $2 \%$ 次氯酸钠处理 $15 \mathrm{~min}$ 后经无菌水冲洗两 遍, 转移到含 $0.6 \%$ 琼脂、 $4 \%$ 蔗糖的 MS 培养基中萌 发。当下胚轴生长至约 $1 \mathrm{~cm}$ 长时, 剪切下胚轴, 使 其长度不超过 $0.5 \mathrm{~cm}$, 然后转移到愈伤组织诱导培 养基 (含 $1 \mathrm{mg} \cdot \mathrm{L}^{-1} 2$, 4-dichlorophenoxyacetic acid （2,4-D）、0.7\% 琼脂、4\%蔗糖的 MS 培养基, $\mathrm{pH} 5.7$ ) 中诱导愈伤组织。将得到的愈伤组织在继代培养基 (含 $0.5 \mathrm{mg} \cdot \mathrm{L}^{-1} 2,4-\mathrm{D}, 0.7 \%$ 琼脂, $4 \%$ 蔗糖的 MS 培 养基, $\mathrm{pH} 5.7$ ) 上继代培养 1 次, 继代周期为 $21 \mathrm{~d}$ 。 培养温度 $(25 \pm 2){ }^{\circ} \mathrm{C}$, 进行暗培养。

1.3 偏二甲肼对芦苇愈伤组织生长半致死剂量的 测定

将生长良好的体积约 $125 \mathrm{~mm}^{3}$ 的愈伤组织块转 移到篮选培养基 (在继代培养基的基础上添加偏二 甲肼) 上培养, 调整偏二甲肼的浓度分别为 0.815 、

$1.63 、 3.26 、 8.15 、 16.3 、 32.6 、 49.9 、 81.5$ 和 $163 \mathrm{mmol} \cdot$ $\mathrm{L}^{-1}$ 。通过观察愈伤组织的生长状况, 确定偏二甲 肼对愈伤组织生长的半致死剂量。

\section{4 抗性细胞系的管选}

首先选取非褐变的生长良好的芦苇愈伤组织, 分别转移至含 0.815 和 $1.63 \mathrm{mmol} \cdot \mathrm{L}^{-1}$ 偏二甲肼的 篮选培养基中培养。将能够正常生长的抗性愈伤组 织在同样的培养条件下继代 1 次, 然后转移至含有 较高浓度偏二甲肼的篮选培养基中培养。如此逐步 
提高笁选压力, 直至接近半致死剂量。分别在培养 23 和 $43 \mathrm{~d}$ 后按照 Smith (1983) 的方法测定愈伤组织 的相对生长量: 相对生长量 $=\left(W_{1}-W_{0}\right) / W_{0} \times$ $100 \%$ 。其中 $W_{1}$ 和 $W_{0}$ 分别代表培养结束时和开始 时愈伤组织的鲜重。计算相对生长量时, 每种篮选 培养基接种 5 瓶, 再重复 1 次, 结果表示为平均值 \pm 标准方差。

\section{5 植株再生}

挑选生长良好的愈伤组织, 转移到分化培养基 (含有 $0.1 \mathrm{mg} \cdot \mathrm{L}^{-1}$ Kinetin ( KT), $0.01 \mathrm{mg} \cdot \mathrm{L}^{-1}$ 1-naphthyl acetic acid（NAA）、0.7\% 琼脂、 $4 \%$ 蔗糖以 及与相对应的篮选培养基等量的偏二甲肼的 MS 培 养基)中进行培养。培养温度为 $(25 \pm 2)^{\circ} \mathrm{C}$, 每天照 光 $14 \mathrm{~h}$, 光照强度为 $60 \mu \mathrm{E} \cdot \mathrm{m}^{-2} \cdot \mathrm{s}^{-1}$ 。愈伤组织的分 化率表示为可分化出幼苗的愈伤组织块占全部愈伤 组织块数量的百分比。将 $70 \mathrm{~d}$ 龄绿色分化苗转移 到不含激素但含有相应偏二甲肼的 MS 培养基中培 养 $35 \mathrm{~d}$, 再转移至 $1 / 2 \mathrm{MS}$ 培养基中培养 $14 \mathrm{~d}$, 然后 转移到由塑料薄膜封盖的含有 $1 / 2$ 蛭石和 $1 / 2$ 营养 土的花盆中适应生长 $21 \mathrm{~d}$, 最后转移至温室中培养。

\section{2 结果与讨论}

\section{1 愈伤组织的诱导及抗性细胞系的笁选}

约 $1 \mathrm{~cm}$ 长的下胚轴接至愈伤组织诱导培养基 上后, $7 \mathrm{~d}$ 左右即可在切口出现愈伤组织, 诱导率为 100\%。芦苇的愈伤组织能够分泌出很多的粘液状 物质, 通过提高培养基中蔗糖的浓度至 $40 \mathrm{~g} \bullet \mathrm{L}^{-1}$, 并 缩短继代周期为 $21 \mathrm{~d}$ 左右, 可以降低粘液状物质的 量。当用愈伤组织诱导培养基作为继代培养基时, 在以后的诱导愈伤组织分化的实验中发现 $70 \%$ 以 上的分化苗为玻璃苗。在分化培养基中加入偏二甲 肼, 几乎所有的分化苗为玻璃苗。单纯改变分化培 养基中激素的配比没有明显成效，但是降低继代培 养基中的 2,4-D 的浓度可以显著降低玻璃苗的出现 几率。因此, 本文中继代培养基中 2,4-D 的浓度降 为 $0.5 \mathrm{mg} \cdot \mathrm{L}^{-1}$ 。

芦苇的愈伤组织转移到含有偏二甲肼的笁选培 养基中后, 生长缓慢。当偏二甲肼的浓度达到 16.3 $\mathrm{mmol} \cdot \mathrm{L}^{-1}$ (1000 ppm) 时, 约一半的愈伤组织死亡, 而且存活下来的抗性愈伤组织块几乎都有局部的褐 变发生, 愈伤组织的增殖几乎没有。由此确定偏二 甲肼对芦苇愈伤组织的半致死剂量为 $16.3 \mathrm{mmol}$ ・ $\mathrm{L}^{-1}$ 左右。由于愈伤组织在篎选培养基中生长缓 慢, 使得继代时间延长, 减缓了篮选速度。因此本试
验首先将愈伤组织转移至含 0.815 和 $1.63 \mathrm{mmol} \cdot$ $\mathrm{L}^{-1}$ 偏二甲肼的两种篎选培养基中进行篎选, 然后 逐渐提高笁选压力。经过约 $3 \sim 6$ 次继代培养, 得到 可在含 1.63 .3 .26 和 $8.15 \mathrm{mmol} \cdot \mathrm{L}^{-1}$ 偏二甲肼的篎 选培养基上正常生长的抗性细胞系。抗性细胞系在 分别培养 $23 \mathrm{~d}$ 和 $43 \mathrm{~d}$ 后的相对生长量如图 1 所示。

23 和 $43 \mathrm{~d}$ 后, 生长在含 $1.63 \mathrm{mmol} \cdot \mathrm{L}^{-1}$ 偏二甲 肼的筛选培养基上的抗性细胞系的相对生长量分别 为对照的 $90.4 \%$ 和 $95.6 \%$ 。生长在含 $3.26 \mathrm{mmol}$ • $\mathrm{L}^{-1}$ 偏二甲肼的篎选培养基上的抗性细胞系的相对 生长量分别为对照的 $84.3 \%$ 和 $91.7 \%$ 。而生长在 含 $8.15 \mathrm{mmol} \cdot \mathrm{L}^{-1}$ 偏二甲肼的篮选培养基上的抗性 细胞系的相对生长量均不到对照的一半 (分别为 $43.4 \%$ 和 $46.8 \%$ ), 与其它两种处理的结果差异显 著, 说明该浓度的偏二甲肼对愈伤组织的生长抑制 作用明显。从图 1 中还可以看出，随着培养时间的 延长, 愈伤组织的生长速率下降, 其中未经偏二甲肼 胁迫的愈伤组织的下降程度最大, 这可能与培养基 中养分的消耗有关。

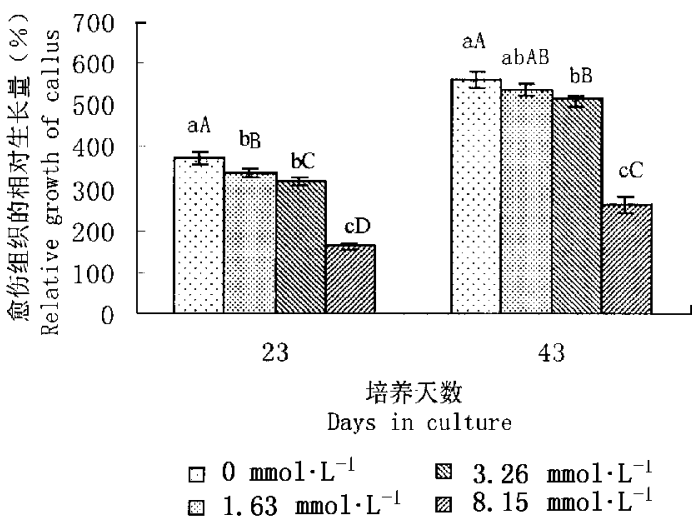

图 1 培养 23 和 $43 \mathrm{~d}$ 后愈伤组织在篮选培养基上的相对生长量

Fig. 1 Relative growth of callus at the end of 23-day and 43-day subculture in screening medium respectively

柱形图上方的误差棒代表正负标准方差 $(n=5)$, 相同的大 (小) 写字母表示不同处理间在同一培养时期内的值在 1\%(5\%) 水平上 不存在显著性差异 Vertical bars indicate the standard devirations $(n=$ 5). Within the same subculture interval, treatments marked with the same capital (small) letter are not significantly different at the level of $1 \%(5 \%)$

\section{2 植株再生}

将在含有 $1.63 、 3.26$ 和 $8.15 \mathrm{mmol} \cdot \mathrm{L}^{-1}$ 偏二甲 肼的篮选培养基上得到的抗性细胞系分别转移到对 应的分化培养基( 以 $0.1 \mathrm{~g} \cdot \mathrm{L}^{-1} \mathrm{KT} 、 0.01 \mathrm{~g} \cdot \mathrm{L}^{-1} \mathrm{NAA}$ 取代篎选培养基中的 $0.5 \mathrm{~g} \bullet \mathrm{L}^{-1} 2,4-\mathrm{D}$, 保持偏二甲 肼的浓度不变)上诱导分化, $14 \mathrm{~d}$ 以后前两种分化培 养基上的愈伤组织开始出现芽点和不定根, 但是在 含 $8.15 \mathrm{mmol} \cdot \mathrm{L}^{-1}$ 偏二甲肼的篮选培养基上得到的 
抗性细胞系至今不能在其分化培养基上分化。不同 抗性愈伤组织的再生频率如表 1 所示。可以看出偏 二甲肼对愈伤组织分化的不良影响强于对愈伤组织 生长的影响。在试验中还发现, 如果将在含 8.15 $\mathrm{mmol} \cdot \mathrm{L}^{-1}$ 偏二甲肼的笁选培养基上得到的抗性细 胞系转移到含 $3.26 \mathrm{mmol} \cdot \mathrm{L}^{-1}$ 偏二甲肼的分化培养 基中时, 可以出现分化苗, 进一步表明偏二甲肼对愈 伤组织的分化有较大的影响。实验中曾以 $0.1 \mathrm{~g}$ $\mathrm{L}^{-1}$ 6-Benzylaminopurine (6-BA) 和 $0.02 \mathrm{~g} \bullet \mathrm{L}^{-1} \mathrm{NAA}$ 取 代分化培养基中的 $0.1 \mathrm{~g} \cdot \mathrm{L}^{-1} \mathrm{KT}$ 和 $0.01 \mathrm{~g} \cdot \mathrm{L}^{-1}$ $\mathrm{NAA}$, 对愈伤组织的分化率以及玻璃苗的出现率的 影响没有可见的差别。在含有 $1.637 \mathrm{mmol} \cdot \mathrm{L}^{-1}$ 偏二 甲肼的培养基篎选到的绿色再生苗生长至约 $5 \mathrm{~cm}$ 长时, 转移到不含激素但含有相应浓度的偏二甲肼 的 MS 培养基中继续培养 $35 \mathrm{~d}$ 左右, 强化根的生长, 然后开始移栽驯化。35 d 以后, $70 \%$ 以上的分化苗 可以成功移栽到温室中。

表 1 不同抗性愈伤组织的再生频率

Table 1 Regeneration rate of resistant callus

\begin{tabular}{ccc}
\hline $\begin{array}{c}\text { 偏二甲肼的浓度 } \\
\begin{array}{c}\text { Concentration of UDMH } \\
\left(\mathrm{mmol} \cdot \mathrm{L}^{-1}\right)\end{array}\end{array}$ & $\begin{array}{c}\text { 再生频率 } \\
\text { Regeneration } \\
\text { rate }(\%)\end{array}$ & $\begin{array}{c}\text { 每块愈伤组织的 } \\
\text { 平均芽点发生数 } \\
\text { Average number of } \\
\text { shoots on a single callus }\end{array}$ \\
\hline 0 & 88 & 9 \\
1.63 & 55 & 4 \\
3.26 & 42 & 3 \\
8.15 & 0 & 0 \\
\hline
\end{tabular}

经过大量篮选和反复继代, 克服了植株再生时 玻璃苗较多的难题, 首次得到了可以在含有 1.63 和 $3.26 \mathrm{mmol} \cdot \mathrm{L}^{-1}$ 偏二甲肼的培养基中生长良好的芦 苇再生苗, 并已将一部分最先得到的可耐受 1.63 $\mathrm{mmol} \cdot \mathrm{L}^{-1}$ 偏二甲肼的分化苗成功地转移到了温室中。 若在温室中大量繁殖得到的芦苇变异体, 将为在人工 湿地系统中的应用提供较为理想的工程植株。

$$
\text { 参 考 文 献 }
$$

Brix H (1989). The use of aquatic macrophytes in water pollution control. Journal of the Human Environment Research and Management, 18(2), $100-107$.

Ernst H, Rittinghausen S, Wahnschaffe U, Mohr U (1987). Induction of malignant peripheral nerve sheath tumors in European hamsters with 1,1-dimethylhydrazine (UDMH). Cancer Letter, $35,303-311$.

Frazier DE Jr, Tarr MJ, Olsen RG (1991). The in vitro and in vivo effects of 1,1-dimethylhydrazine (UDMH) on murine lympho- cyte subsets and IA antigen expression. Immunopharmacol Immunotoxicol, 13, $25-46$.

Gong BA(宫宝安), Xu XF(徐秀峰), Guo BD(郭保东), Zou DF (邹德峰), Cong PX (丛培学), Wang JG(王建国) (1994). Removal of UDMH from waste by catalytic oxidation. Journal of Yantai University (Natural Science and Engineering) (烟台大学 学报 (自然科学与工程版)), 3,43-46. (in Chinese with English abstract)

Lunn G, Sansone EB (1994). Oxidation of 1,1-dimethylhydrazine (UDMH) in aqueous solution with air and hydrogen peroxide. Chemosphere, 29, 1577 - 1590 .

Meng XH (孟晓红), Wu WE (吴婉娥), Fu CR (傅超然) (2000) . Unsuynnetrical dimethyl-hybrazine pollution and evaluation of its control method. Yunnan Environmeatal Science (云南 环境科学) , 19,165-168. (in Chinese with English abstract)

Smith RH, Bhaskaran S, Schertz K (1983). Sorghum plant regeneration from aluminum selection media. Plant Cell Reproduction, $2,129-132$.

Sun G, Gray KR, Biddlestone AJ, Cooper DJ (1999). Treatment of agricultural wastewater in a combined tidal flow-downflow reed bed system. Water Science and Technology, 40, 139 - 145.

Ye ZH, Baker AJM, Wong MH, Willis AJ (1997). Zinc, lead and cadmium tolerance, uptake and accumulation by the common reed, Phragmites australis (Cav.) Trin. ex Steudel. Annals of Botany, 80, $363-370$.

Yue MX(岳茂兴), Peng RY (彭瑞云), Wang ZG (王正国), Wang DW (王德文), Yang ZH (杨志焕), Yang HM (杨鹤 鸣), Xia YD(夏亚东), Gao YB(高亚兵), Cui XM(崔雪 梅), Li JZ(李建忠), Li Y (李炎) (2004). Intoxication induced by unsymmetrical dimethylhydrazine: characteristics and mechanism of its acute and chronic injuries. Chinese Critieal Care Medicine (中国危重病急救医学), 16, 740-742. ( in Chinese with English abstract)

Zelnick SD, Mattie DR, Stepaniak PC (2003). Occupational exposure to hydrazines: treatment of acute central nervous system toxicity. Aviation Space Environmental Medcine, 74, 1285 - 1291.

Zhang CL(张承烈), Chen GC(陈国仓) (1991). Study on gas exchange characteristics of Phragmites communis Trin. in different ecotypes of dry desert of the Hexi corridor. Acta Ecologica Sinica (生态学报), 11,250-254. (in Chinese with English abstract)

Zhang JD (张家得) (1993). Detection and Protection of Rocket Propellant Fuel and Prevention of Their Pollution (火箭推进剂 的检测与防护及其污染治理措施). National University of Defense Technology Press, Changsha,779-803. (in Chinese) Zhu HB(朱红兵), Zhang T(张统), Sha CL(沙聪亮), Li TD (李铁栋), Jiang Y (蒋义) (2003). Influence analysis of rocket fuels to the groundwater of launch base. Water and Wastewater Engineering (给水排水), 29,37 - 39. ( in Chinese with English abstract) 